\title{
Time Evolution of Localized Waves in Non thermal Distributed Plasmas: Considering Dissipation Effects
}

\author{
Alidad Askari* and Rohollah Soleimani \\ Department of Physics, Hormozgan University, Po. Box 3995 Bandar Abbas, Iran; askari@hormozgan.ac.ir, \\ alidadaskari@gmail.com, rohollahsolimani@gmail.com
}

\begin{abstract}
Recent observations show the existence of high energy electrons in various astrophysical plasmas which their distribution functions are highly non thermal. The presence of dust ion acoustic localized waves in plasmas which contain non thermal electrons and ions with kinematic viscosity along with stationary charged dust is investigated. Solitary shock waves are derived as solutions of nonlinear wave equation of the medium using the reductive perturbation method by employing suitable stretching coordinates. Both rarefactive and compressive shock profiles are created in the medium according to the initial values of the medium parameters. We report that increasing the electron number density makes a growth in the amplitude of negative shock waves while it decreases the amplitude of positive shock profile. Some behavior of observed transience in the plasma waves can be explained using the presented results.
\end{abstract}

Keywords: Dusty Plasmas, Dust-Ion-Acoustic Solitary Waves, Localized Waves, Non Thermal Distribution, Shock Waves, Viscosity

\section{Introduction}

In recent years, dusty plasmas have attracted a great interest due to their significance in astrophysics and upper parts of the atmosphere (Planetary Rings, Comets and Magnetosphere) and also in the lower components of the Earth's ionosphere ${ }^{1-4}$. In this medium we have charged dust particles which have either negative or positive charge depending on the plasma environments that surrounds them ${ }^{5-7}$. The low-frequency Dust-ion-acoustic (DIA) wave is one of the main electrostatic dust-associated waves that exist in dusty plasmas without magnetic field. It was first pointed out by Shukla and Silin ${ }^{8}$ that because of the inequality $\mathrm{n}_{\mathrm{e} 0}<<\mathrm{n}_{\mathrm{i} 0}$ and conservation of equilibrium charge density $\mathrm{n}_{\mathrm{e} 0}+\mathrm{Z}_{\mathrm{d}} \mathrm{n}_{\mathrm{d} 0}=\mathrm{n}_{\mathrm{i} 0}$ [ where $\mathrm{Z}_{\mathrm{d}}$ is the number of electrons residing onto the dust grain surface, $e$ is the value of the charge of the electron and $n_{d 0}, n_{i 0}$ and $\mathrm{n}_{\mathrm{e} 0}$ are, respectively, dust, ion and electron number density at equilibrium] a negatively charged static dusty plasma could tolerate low-frequency Dust-ion-acoustic (DIA) waves (DIAWs) with the condition that phase velocity should be very smaller (larger) than the thermal speed of electron (ion). It should be pointed out that DIAWs have also been seen in laboratory experiments ${ }^{9,10}$. Also, it should be noted that the dynamics of DIA waves is significantly different than the dust-acoustic waves in which it is important to consider the dust dynamics in our model because the inertia is supplied by the mass of the dust particle and the restoring force is provided by the pressures of inertia less electrons and ions ${ }^{11-13}$, i.e.,. Different linear and nonlinear properties of dust ion-acoustic waves have gained a large interest in comprehension of the basic features of localized electrostatic perturbations in astrophysics and laboratory dusty plasmas ${ }^{14-22}$. From both theoretical and experimental point of view, it has been shown that a dusty plasma which is weakly coupled has two novel Eigen modes, i.e. dust-acoustic mode ${ }^{12,13}$ and the dust-ion-acoustic mode ${ }^{8-10}$. The main reasons for starting

${ }^{*}$ Author for correspondence 
dissipation which is the cause for the formation of shock structures in plasmas are as follows: the Landau damping, kinematic viscosity between different parts of Plasma, Ion-neutral and Dust-neutral collisions. The existence of high energy electrons in various astrophysical plasmas has been confirmed by many observations and measurements show that their distribution functions is highly non thermal. This non thermal feature is turned out to be a very common in astrophysical plasmas in which it is expected that coherent nonlinear waves and structures play a major role. Non thermal distributions might spread isotropically in velocity space or move with respect to the background plasma and also their existence has been established by various observations ${ }^{23-26}$. Viking spacecraft ${ }^{27}$ and Freja satellite ${ }^{28}$ have confirmed the presence of electrostatic solitary structures in the magnetosphere with density depressions. Inspired by these observations, Cairns et al. ${ }^{29}$ showed that the existence of a non thermal population of electrons may alter the properties of ion sound solitary structures and permit the presence of rarefactive ionacoustic solitary structures very similar to those seen by Freja and Viking. Some recent articles have concentrated on the consequences of non thermality of particles on various types of linear and nonlinear collective process$\mathrm{es}^{30-39}$. Our main aim in the present paper is therefore to survey the existence of dust ion-acoustic shock waves in plasmas having non thermal electron and ion kinematic viscosities along with stationary charged dust. Thus, it is worthwhile to give a first theoretical work to study DIA shock waves in a nonthermal dusty plasma. The present work is structured as follows. In Section 2 we present the basic equations that govern the electronegative plasma system. The basic features of the dust ion-acoustic shock waves are investigated in Section 3, while those of the dust ion-acoustic shock waves are investigated in Section 4. Finally, we summarize our results in Section 5.

\section{Basic Equation}

The nonlinear dynamics of Dust-ion-acoustic waves with the condition that phase velocity is much smaller (larger) than the electron (ion) thermal velocity is ruled by:

$$
\begin{gathered}
\frac{\partial n}{\partial t}+\frac{\partial(n u)}{\partial x}=0 \\
\frac{\partial u}{\partial t}+u \frac{\partial u}{\partial x}=-\frac{\partial \phi}{\partial x}+\eta \frac{\partial^{2} u}{\partial x^{2}}
\end{gathered}
$$

$$
\frac{\partial^{2} \phi}{\partial x^{2}}=\mu m_{e}-n+1-\mu
$$

in which $n$ is the ion number density that is normalized by its equilibrium value $n_{0 i} u$ is the ion fluid velocity normalized by $c_{i}=\sqrt{T_{e} / m_{i}}$ is the electrostatic wave potential normalized by $\left(T_{e} / e\right)$, where $T_{e}$ is electron temperature. Also $\eta$ is the viscosity coefficient normalized $m_{i} n_{i 0} \omega_{p i} \lambda_{D m}^{2}$. The time $t$ and the distance $x$ are normalized respectively by the ion plasma frequency $\omega_{p i}^{-1}=\sqrt{m_{i} / 4 \pi n_{i 0} e^{2}}$ and the Debye radius $\lambda_{D m}=\sqrt{T_{e} / 4 \pi n_{i 0} e^{2}}$. We have denoted $\mu=n_{e 0} / n_{i 0}$.

We use the non-thermal electron distribution of Cairns et al. ${ }^{29}$ which is a general class of the high energy electron distribution to model the electron population in the presence of fast or non-thermal particles. Therefore, we use

$$
n_{e}=\left(1-\beta \phi+\beta \phi^{2}\right) e^{\phi}
$$

in which $\beta=\frac{4 a}{1+3 \alpha}$. $\alpha$ Is a parameter that characterizes the distribution of nonthermal energetic electrons and determines the degree of nonthermality.

\section{Burgers Equation}

We develop a theory of DIA waves which is weakly nonlinear with small but finite amplitude to derive the Burgers equation for a dusty plasma model under study that results a scaling of the independent variables ${ }^{40}$.

$$
\tau=\varepsilon^{2} t, \xi=\varepsilon(x-\lambda t)
$$

which $\varepsilon$ is a small parameter and $\lambda$ is phase speed of the wave. Now we expand each variable in powers of $\varepsilon$ as

$$
\begin{aligned}
& n=1+\varepsilon n_{1}+\varepsilon n_{2}+\ldots \\
& u=\varepsilon u_{1}+\varepsilon u_{2}+\ldots \\
& \phi=\varepsilon \phi_{1}+\varepsilon \phi_{2}+\ldots
\end{aligned}
$$

Now, we replace the stretching Equation (5) and the Expansions (6) into our governing Equations (1-4) and collect the terms in the different powers of $\varepsilon$, to the lowest-order in $\varepsilon$, we obtain

$$
\begin{aligned}
\lambda^{2} n_{i}^{(1)} & =\phi_{i}^{(1)} \\
\lambda u_{i}^{(1)} & =\phi_{i}^{(1)}
\end{aligned}
$$

and 


$$
\lambda=\sqrt{\frac{1}{\mu(1-\beta)}}
$$

and for the higher orders of $\varepsilon$, we have

$$
\begin{gathered}
-\lambda \frac{\partial n_{2}}{\partial \xi}+\frac{\partial n_{1}}{\partial \tau}+\frac{\partial\left(n_{1} u_{1}\right)}{\partial \xi}+\frac{\partial u_{2}}{\partial \xi}=0, \\
-\lambda \frac{\partial u_{2}}{\partial \xi}+\frac{\partial u_{1}}{\partial \tau}+\frac{\partial \phi_{2}}{\partial \xi}+u_{1} \frac{\partial u_{1}}{\partial \xi}-\eta \frac{\partial^{2} u_{1}}{\partial \xi^{2}}=0, \\
\frac{\partial^{2} \phi_{1}}{\partial \xi^{2}}=\frac{1}{2} \mu \phi_{1}^{2}+\mu(1-\beta) \phi_{1}^{2}-n_{2}
\end{gathered}
$$

Now we derive the Berger Equation from (7) and (10) as

$$
\frac{\partial \phi_{1}}{\partial \tau}+\mathrm{A} \phi_{1} \frac{\partial \phi_{1}}{\partial \xi}-C \frac{\partial^{2} \phi_{1}}{\partial \xi^{3}}=0
$$

where

$$
A=\frac{\lambda^{3}}{2}\left[\frac{3}{\lambda^{4}}-\mu\right]
$$

and

$$
C=\frac{\eta}{2}
$$

Where $\mathrm{A}$ and $\mathrm{C}$ are respectively the nonlinearity and dissipative coefficients.

\section{Numerical Results and Discussion}

Equation (11) is the burgers equation which describes dynamics of the nonlinear Dust ion-acoustic shock wave containing the electron nonthermality effect and the kinematic viscosity between the plasma components. Equation (11) describes a travelling wave without deformation at constant velocity $u$ and possesses a stationary shock wave solution. By introducing $\zeta=\zeta-u \tau^{\prime}$ and $\tau=\tau^{\prime}$, under the steady state condition, Equation (11) takes the following form ${ }^{41}$.

$$
-U_{0} \frac{\partial \varphi_{1}}{\partial \zeta}+A \varphi_{1} \frac{\partial \varphi_{1}}{\partial \zeta}=C \frac{\partial^{2} \varphi_{1}}{\partial \zeta^{2}}
$$

It can be easily shown that the above equation describes the shock waves. The shock wave speed is connected to the extreme values $\phi_{1}(-\infty)$ and $\phi_{1}(\infty)$ through $\phi_{1}(\infty)-\phi_{1}(-\infty)=2 u / A$. So under the conditions that $\phi_{1}$ is limited at $\zeta \rightarrow \pm \infty$, the shock wave solution of (12) is in the following form

$$
\varphi_{1}=\varphi_{m}\left[1-\tanh \left(\frac{\zeta}{W}\right)\right],
$$

Where the thickness and amplitude of the shock wave are defined as $W=2 C / u$ and $\phi_{m}=u / A$, respectively. It must be noted that the mutual balance between dissipation and nonlinearity leads to formation of this type of shock solution. It is clear that an increase in $u$ enhances the height of shock wave, and reduces its width. It is possible to see that the amplitude of shock waves can take both positive and negative sign, through Equation (14). The nonlinear coefficient $A$ tends to zero as $\beta \rightarrow \beta_{c}=1-1 / \sqrt{3 \mu}$, thus for $\beta<\beta_{c}\left(\beta>\beta_{c}\right)$ we have positive (negative) DIA shock wave. This result is in agreement with ${ }^{22}$. In Figure 1, we have shown how the critical value of nonthermal parameter $\left(\beta_{c}\right)$ varies with relative density $(\mu)$. It is possible to observe that the result of growing $\mu$ is to higher the critical nonthermal parameter $\beta_{c}$ which above this parameter only rarefactive Dust-ion-acoustic shock waves are allowed. Note that for $\mu<0.33$, only negative polarity of DIA shock can be created and in this situation, nonthermality doesn't have effect on the shock polarity. It is obvious that for other values of $\mu(\mu>1 / 3)$, polarity of shock wave depend sensitively to nonthermality. Also, a similar behavior discussed by Baluku et al. ${ }^{42}$ and Yasmin et $\mathrm{al}^{40}$.

To investigate the influence of the dissipation and electron nonthermality effects on the fundamental features of dust-ion-acoustic shock waves in this model, the

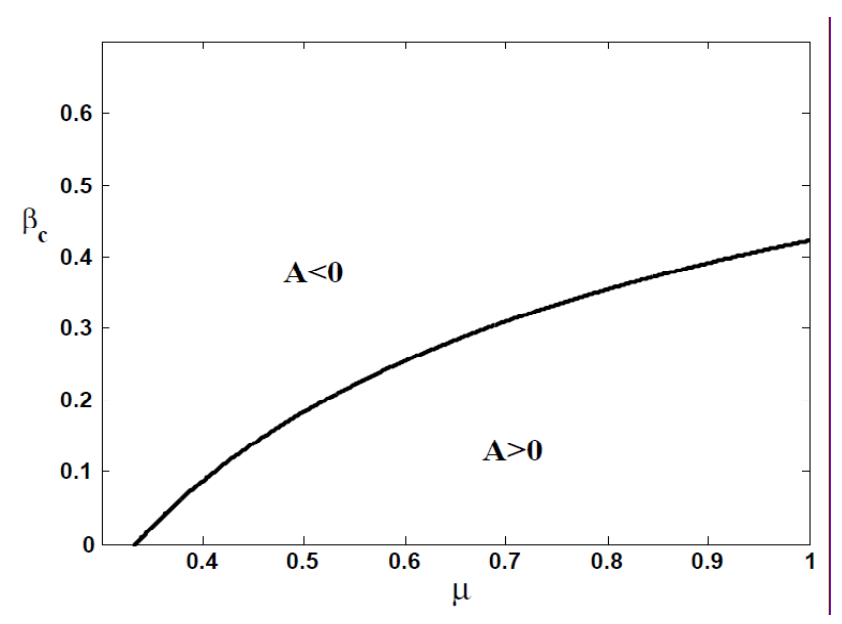

Figure 1. Plot of Critical Nonthermal Parameter $\left(\beta_{c}\right)$ with Relative Density $(\mu)$ (when a becomes zero). 
solutions of shock waves are found numerically. Thus, this model supports excitation of DIA shock waves with both positive and negative polarities. The results are displayed in Figures 1-3.

The dependency of DIA shock structure on the electron nonthermality is investigated in Figure 2, for various values of the electron number density. It is clear that for positive shock wave an increase in $\beta$ enhances the amplitude of the shock structure, while the negative shock wave displays an inverse treatment. This behavior means that departure of electrons from Maxwellian treatment $(\beta=0)$ leads to appearance of positive (negative) DIA shock waves with larger (smaller) amplitude.

In order to investigate the effect of dissipation on the dust ion-acoustic shock structure, we have depicted the

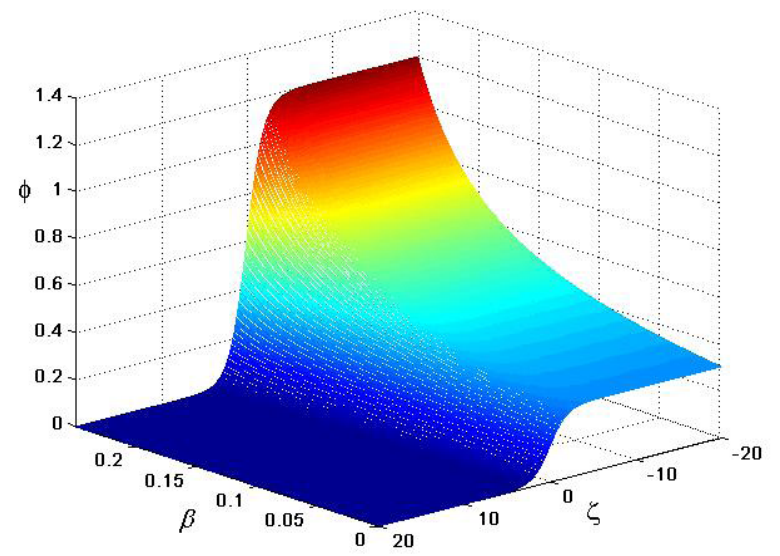

(a)

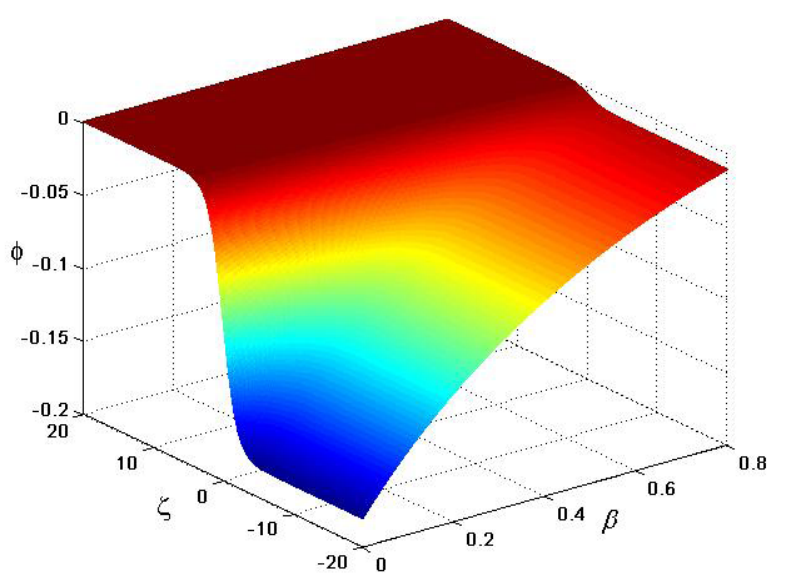

(b)

Figure 2. (a) The Electrostatic Potential $\phi$ as a function of $\zeta$ and $\beta$ with $\mu=0.7, \eta=0.2$ and $\mathrm{u}=0.1$. (b) The Electrostatic Potential $\phi$ as a function of $\zeta$ and $\beta$ with $\mu=0.1, \eta=0.2$ and $u=0.1$.

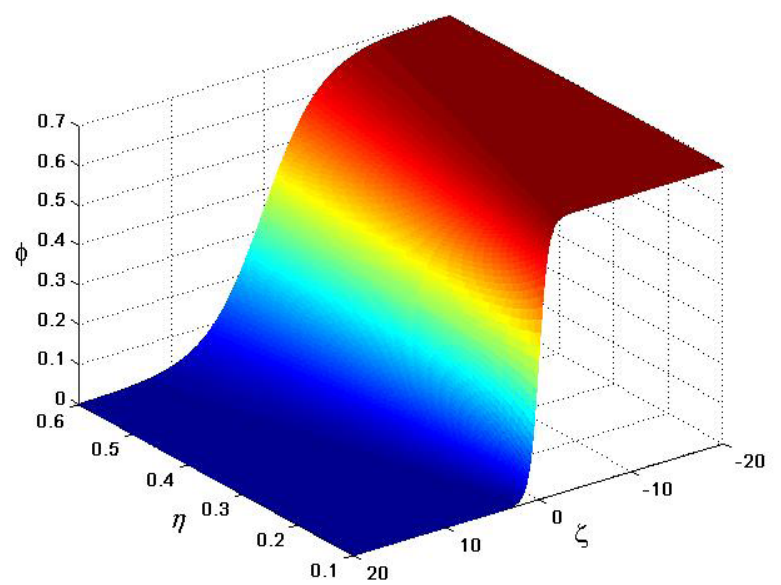

(a)

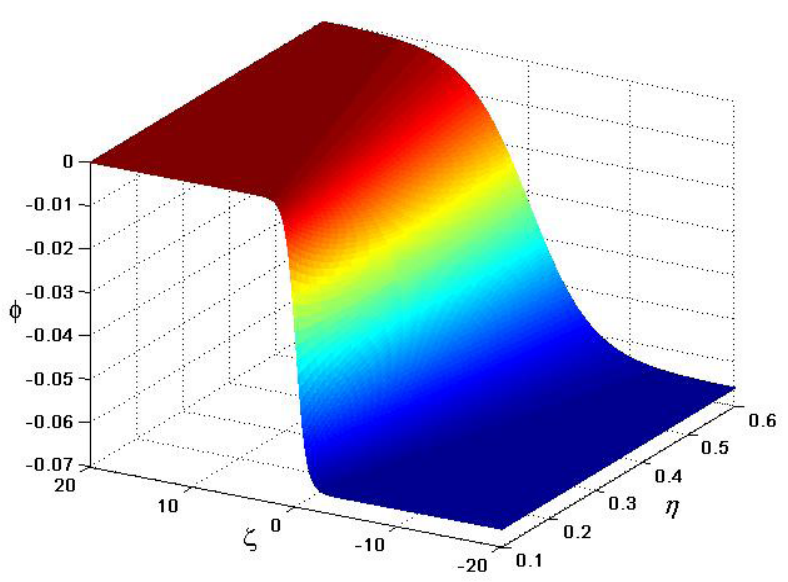

(b)

Figure 3. (a) The Electrostatic Potential $\phi$ as a function of $\zeta$ and $\eta$ with $\mu=0.7, \beta=0.2$ and $\mu=0.1$. (b) The Electrostatic Potential $\phi$ as a function of $\zeta$ and $\eta$ with $\mu=$ $0.1, \beta=0.4$ and $u=0.1$.

behavior of the electrostatic potential with respect to $\zeta$ and $\eta$ in Figure 3 for two set of parameters $\mu=0.7$, $\beta=0.2$ and $\mu=0.1, \beta=0.4$. The first set leads to positive shock and for second set a negative shock structure appears. We see that the thickness of shock wave increases with dissipation term. This is consistent with the result of Yasmin et $\mathrm{a}^{40}$. Figure 4 indicates variation of the electrostatic potential with respect to $\zeta$ and $\mu$, for $\beta=0.1$ and $\beta=0.4$, respectively, in Figures $4 \mathrm{a}$ and $4 \mathrm{~b}$. This figure indicates that a growth of the electron number density induces increases of the amplitude of negative shock wave (Figure $4 \mathrm{a}$ ) whereas it decreases the amplitude of positive shock wave (Figure $4 b$ ). This result is in agreement with ${ }^{40,43}$. 


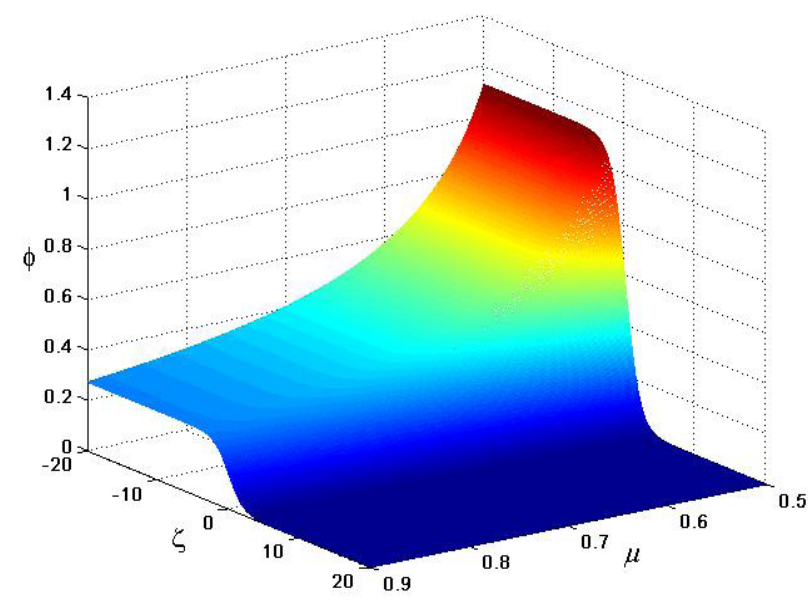

(a)

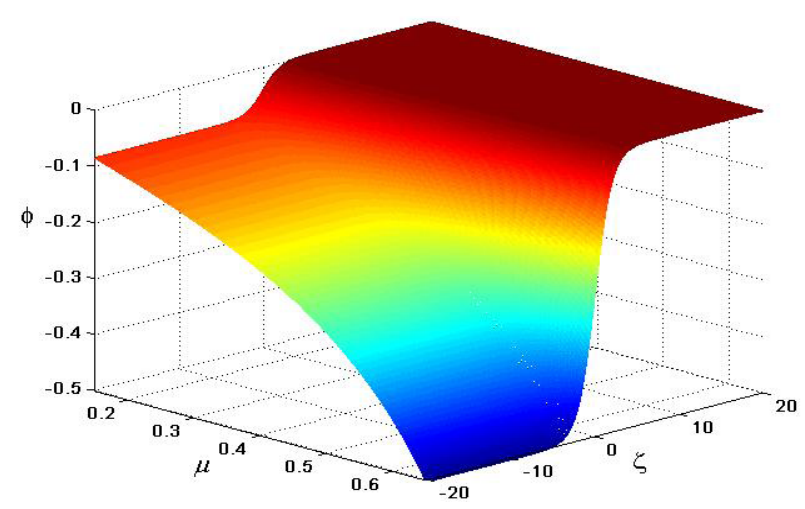

(b)

Figure 4. (a) The Electrostatic Potential $\phi$ as a function of $\zeta$ and $\mu$ with $\beta=0.1, \eta=0.2$ and $u=0.1$. (b) The Electrostatic Potential $\phi$ as a function of $\zeta$ and $\mu$ with $\beta=0.4, \eta=0.2$ and $u=0.1$.

\section{Conclusions}

In this manuscript, we have studied the nonlinear dynamics of DIA shock waves in dusty plasma constructed from Inertial Ions, Nonthermal Electrons, and Stationary dust particles. We included the dissipation by considering kinematic viscosity between the various parts of the plasma. In addition, we derived the Burgers equation for the propagation of DIA shock waves by applying the reductive perturbation method, for the propagation of DIA shock waves. The present model supports the presence of both positive and negative DIA shock structures. We have shown that the departure of electrons from thermodynamics equilibrium leads to appearance of positive (negative) Dust ionacoustic shock waves with larger (smaller) amplitude.
It is shown that a growth in dissipation term leads to increase of the shock wave thickness. It is also observed that a growth in the electron number density leads to an increase of the amplitude of negative shock waves whereas it decreases the amplitude of positive shock waves. This study would be helpful in comprehension of the basic nonlinear properties of the dust ionacoustic shock wave that might appear in astrophysical dusty plasmas, particularly those containing the nonthermal components, the ionosphere ${ }^{44}$ and the auroral acceleration regions ${ }^{45}$.

\section{References}

1. Mendis DA, Horanyi M. Dust-plasma Interactions in the Cometary Environment. Cometary Plasma Processes; American Geophysical Union; Washington: DC. 1991. p. 17.

2. Pieper JB, Goree J. Dispersion of Plasma Dust Acoustic Waves in the Strong-Coupling Regime. Phys. Rev. Lett. 1996; 77:3137.

3. Shukla PK. Dust-ion-Acoustic Shocks and Holes. Phys. Plasmas. 2000; 7:1044.

4 Rosenberg M, Merlino RL. On-acoustic Instability in a Dusty Negative Ion Plasma. Planet. Space Sci. 2007; 55:1464.

5. Goertz CK. Dusty Plasmas in the Solar System. Rev. Geophys. 1989; 27:271. Doi: 10. 1029/RG027i002p00271.

6. Shukla PK, Mamun AA. Introduction to Dusty Plasma Physics. IOP; Bristol. 2002.

7. Shukla PK, Eliasson B. Fundamentals of Dust-Plasma Interactions. Rev. Mod. Phys. 2009; 81:25.

8. Shukla PK, Silin VP. Dust ion-Acoustic Wave. Phys. Scripta. 1992; 45:508.

9. Merlino RL, Goree J. Dusty Plasmas in the Laboratory, Industry, and Space. Phys. Today. 2004; 57: 32.

10. Barkan A, Angelo ND, Merlino RL. Experiments in IonAcoustic Waves in Dusty Plasmas. Planet. Space Sci. 1996; 44:239.

11. Shukla PK, Rosenberg M. Boundary Effects on Dust-IonAcoustic and Dust-Acoustic Waves in Collisional Dusty Plasmas. Phys Plasmas. 1999; 6:1038.

12. Rao NN, Shukla PK, Yu Y. Dust-acoustic waves in Dusty Plasmas Original Research Article. Planet Space Sci. 1990; 38:543-46.

13. Barkan A, Merlino RL, Angelo ND. Laboratory Observation of the Dust acoustic Wave Mode. Phys Plasmas. 1995; 2:3563.

14. Moslem WM, El-Taibany WF, El-Shewy EK, El-Shamy EF. Dust-Ion-Acoustic Solutions with transverse perturbation. Phys Plasmas. 2005; 12:052318. 
15 Shukla PK, Mamun AA. Solutions, shocks and vortices in dusty plasmas. New J. Phys. 2003; 5:17.

16. Bharuthram R, Shukla PK. Large Amplitude Ion-Acoustic Solutions in Dusty plasma Original Research Article. Planet Space Sci. 1992; 40:973-77.

17. Nakamura Y, Bailung H, Shukla PK. Observation of IonAcoustic Shocks in Dusty Plasma. Phys. Rev. Lett. 1999; $83: 1602$

18. Nakamura Y, Sharma A. Observation of Ion-Acoustic Solitary Waves in a Dusty Plasma. Phys. Plasmas. 2001; 8:3921-6.

19. Mamun AA, Shukla PK. Cylindrical and Spherical Dust Ion-Acoustic Solitary Waves. Phys. Plasmas. 2002; 9:1468.

20. Mamun AA, Shukla PK. The role of dust charge fluctuations on nonlinear dust-ion-acoustic Waves. IEEE Transaction Plasma Science. 2002; 30:720.

21. Mamun AA, Shukla PK, Nonlinear Waves and Structures in Dusty Plasmas. Plasma Phys Controlled Fusion. 2005; 47:A1.

22. Berbri A, Tribeche M. Weakly Nonlinear Dust-IonAcoustic Shock Waves in a Dusty Plasma with Nonthermal Electrons. Physics Plasmas. 2009; 16:053701.

23. Goldman MV, Oppenheim MM, Newman DL. Theory of Localized Bipolar Wave-Structures and Nonthermal Particle Distributions in the Auroral Ionosphere. Nonlinear Processes in Geophys. 1999; 6:221-8.

24. Feldman WC, Anderson SJ, Bame SJ, Gary SP, Gosling JT, McComas DJ, Thomsen MF, Paschmann G, Hoppe MM. Electron velocity distributions near the Earth's bow shock. Geophys J Res. 1983; 88(A1):96-110. Doi: 10.1029/ JA088iA01p00096.

25. Lundlin R, Zakharov A, Pellinen R, Borg H, Hultqvist B, Pissarenko N, Dubinin EM, Barabash SW, Liede I, Koskinen H. First Measurements of the Ionosphere Plasma Escape from Mars. Nature London. 1989; 341:609-12.

26. Futaana Y, Machida S, Saito Y, Matsuoka AA, Hayakawa HH. Moon-Related Nonthermal Ions Observed by Nozomi: Species, Sources, and Generation Mechanisms. Geophys J Res. 2003; 108:151. Doi: 10.1029/2002JA009366.

27. Bostrom R. Observations of Weak Double Layers on Auroral Field Lines. IEEE Trans. Plasma Sci. 1992; 20:756.

28. Dovner PO, Eriksson AI, Bostrom R, Holback B. Freja Multiprobe Observations of Electrostatic Solitary Structures. Geophys Res. Lett.1994; 21:1827-34.

29. Cairns RA, Mamun AA, Bingham R, Boström R, Dendy RO, Nairn C MC, Shukla PK, Electrostatic Solitary Structures in Non-thermal Plasmas. Geophys Res. Lett. 1995; 22(20):2709-12.

30. Zhang LP, Xue JK. Shock Wave in Magnetized Dusty Plasmas with Dust Charging and Nonthermal Ion Effects. Phys Plasmas. 2005; 12:042304.
31. Xue JK, Zhang P. Nonlinear Waves in Nonplanar and Nonuniform Dusty Plasmas. Phys Plasmas. 2006; 13:022104.

32. El-Taibany WF, Kourak I. Modulational instability of dust acoustic waves in dusty plasmas: modulation obliqueness, background ion nonthermality, and dust charging effects. Phys Plasmas. 2006; 13:062302.

33. El-Taibany WF, Wadati M, Sabry R. Nonlinear Dust Acoustic Waves in a nonuniform magnetized complex plasma with nonthermal ions and dust charge variation. Phys Plasmas. 2007; 14:032304.

34. Tribeche M, Amour R. Nonlinear localized dust acoustic waves in a charge varying dusty plasma with nonthermal ions. Phys. Plasmas. 2007; 14:103707.

35. Tribeche M, Boumezoued G. Effect of electron nonthermality on nonlinear electrostatic solitary waves in a charge varying dusty plasma. Phys Plasmas. 2008; 15:053702.

36. Hakimi Pajouh H, Abbasi H. Dust-ion-acoustic solutions in plasmas with non-Maxwellian electron distribution function. Phys. Plasmas. 2008; 15:103705.

37. Alinejad H. Dust ion-acoustic solitary and shock waves in a dusty plasma with non-thermal electrons. Astrophys Space Sci. 2010; 325:209-215.

38. Alinejad H. Dust ion-acoustic solitary and shock waves in a dusty plasma with non-thermal electrons. Astrophys Space Sci. 2010; 327:131-7.

39. Yasmin S, Asaduzzaman M, Mamun AA. Dust-ion-acoustic shock waves in nonextensive dusty plasma. Astrophys Space Sci. 2013; 343:245-50.

40. Shahmansouri M, Alinejad H. Arbitrary amplitude dust ion acoustic solitary waves in a magnetized suprathermal dusty plasma. Phys Plasmas. 2012; 19(12):123701.

41. Baluku TK, Hellberg MA, Kourakis I, Saini NS. Ion acoustic solutions in a plasma with kappa-distributed electrons. Phys Plasmas. 2010; 17:053702.

42. Alinejad H, Shahmansouri M. Low intensity dust-ionacoustic shock waves due to dust charge fluctuation in a nonextensive dusty plasma. Phys Plasmas. 2012; 19:083705.

43. Bremer J, Hoffmann P, Manson AH, Meek CE, Ruster R, Singer W. PMSE observations at three different frequencies in northern Europe during summer 1994. Ann Geophys. 1996; 14:1317-27.

45. Ergun R, Carlson CW, McFadden JP, et al. FAST satellite observations of large-amplitude solitary structures. Geophys Res Lett. 1998; 25:2041-2044. Doi: 10.1029/98GL00636.

46. Shukla PK, Mamun AA, Solitons. Shocks and vortices in dusty plasmas. New J Phys. 2003; 5:17.1-17.37.

47. Ghosh S, Bharuthram R, Khan M, Gupta MR. Instability of dust acoustic wave due to nonthermal ions in a charge varying dusty plasma. Physics of Plasmas.1994; 11(7):3602-09. 